\title{
BMJ Open Ethnicity and outcomes in patients hospitalised with COVID-19 infection in East London: an observational cohort study
}

\author{
Vanessa J Apea, ${ }^{1,2}$ Yize I Wan (D) , , ${ }^{3,4}$ Rageshri Dhairyawan, ${ }^{1,2}$ \\ Zudin A Puthucheary, ${ }^{3,4}$ Rupert M Pearse, ${ }^{3,4}$ Chloe M Orkin, ${ }^{1,2}$ John R Prowle ${ }^{3,4}$
}

To cite: Apea VJ, Wan YI, Dhairyawan R, et al. Ethnicity and outcomes in patients hospitalised with COVID-19 infection in East London: an observational cohort study. BMJ Open 2021;11:e042140. doi:10.1136/ bmjopen-2020-042140

- Prepublication history and additional materials for this paper is available online. To view these files, please visit the journal online (http://dx.doi org/10.1136/bmjopen-2020042140).

VJA and YIW are joint first authors.

$\mathrm{CMO}$ and JRP are joint senior authors.

Received 26 June 2020 Revised 07 November 2020 Accepted 13 November 2020

Check for updates

(C) Author(s) (or their employer(s)) 2021. Re-use permitted under CC BY-NC. No commercial re-use. See rights and permissions. Published by BMJ.

For numbered affiliations see end of article.

Correspondence to

Dr Yize I Wan;

yize.wan@qmul.ac.uk

\section{ABSTRACT}

Objective To describe outcomes within different ethnic groups of a cohort of hospitalised patients with confirmed COVID-19 infection. To quantify and describe the impact of a number of prognostic factors, including frailty and inflammatory markers.

Setting Five acute National Health Service Hospitals in east London.

Design Prospectively defined observational study using registry data.

Participants 1737 patients aged 16 years or over admitted to hospital with confirmed COVID-19 infection between 1 January and 13 May 2020.

Main outcome measures The primary outcome was 30-day mortality from time of first hospital admission with COVID-19 diagnosis during or prior to admission. Secondary outcomes were 90-day mortality, intensive care unit (ICU) admission, ICU and hospital length of stay and type and duration of organ support. Multivariable survival analyses were adjusted for potential confounders.

Results 1737 were included in our analysis of whom 511 had died by day 30 (29\%). 538 (31\%) were from Asian, $340(20 \%)$ black and 707 (40\%) white backgrounds. Compared with white patients, those from minority ethnic backgrounds were younger, with differing comorbidity profiles and less frailty. Asian and black patients were more likely to be admitted to ICU and to receive invasive ventilation (OR 1.54, $(95 \% \mathrm{Cl} 1.06$ to 2.23); $p=0.023$ and OR 1.80 (95\% $\mathrm{Cl} 1.20$ to 2.71$) ; p=0.005$, respectively). After adjustment for age and sex, patients from Asian (HR 1.49 (95\% Cl 1.19 to 1.86); $p<0.001$ ) and black (HR 1.30 (95\% Cl 1.02 to 1.65 ); $p=0.036$ ) backgrounds were more likely to die. These findings persisted across a range of risk factor-adjusted analyses accounting for major comorbidities, obesity, smoking, frailty and $\mathrm{ABO}$ blood group.

Conclusions Patients from Asian and black backgrounds had higher mortality from COVID-19 infection despite controlling for all previously identified confounders and frailty. Higher rates of invasive ventilation indicate greater acute disease severity. Our analyses suggest that patients of Asian and black backgrounds suffered disproportionate rates of premature death from COVID-19.

\section{INTRODUCTION}

The novel SARS-CoV-2, which manifests as COVID-19, has led to a global pandemic. ${ }^{1}$
Strengths and limitations of this study

- This study is one of the most comprehensive studies exploring COVID-19 outcomes in black, Asian and minority ethnic populations so far reported including evaluation of linked comorbid and socioeconomic risk factors.

- This study was conducted in a single region where COVID-19 has had significant impact and thus not confounded by differences in incidence of COVID-19 disease across the UK, regional concentration of minority ethnic groups and regional differences in the time course of the epidemic.

- In addition, we employed a prespecified statistical analysis plan and performed multiple sensitivity analyses to test the robustness of our findings.

- In line with the vast majority of published COVID-19 analyses, we only included proven COVID-19 cases, therefore, suspected diagnoses should be considered in future studies, particularly those occurring outside of hospitals.

- Despite its size, our study lacked the power to assess a more detailed ethnicity breakdown and like many datasets, may not reflect the vast heterogeneity within ethnic categories (such as Bangladeshi, Pakistani, black African or black Caribbean).

Older age, male sex, obesity and pre-existing health conditions such as diabetes and hypertension have all been identified as risk factors for poor outcomes. ${ }^{2-4}$ A disproportionate impact of disease severity and death on people from black, Asian and minority ethnic (BAME) backgrounds has been reported, though not consistently. The UK Intensive Care National Audit and Research Centre (ICNARC) noted that while BAME groups only make up $14 \%$ of the UK population, they comprised $33 \%$ of COVID-19 patients on intensive care units (ICUs) ${ }^{5}$ The degree of this excess risk also appears to differ across, and within, these heterogeneous ethnic groups. In the UK, recent analyses of data from the Office of 
National Statistics and National Health Service (NHS) England described 2.5-fold to 4.3-fold greater COVID-19 mortality rates, compared with white groups, across a range of black and South Asian ethnic groups. ${ }^{6}$ Whether this adverse association is driven by underlying comorbid disease, socioeconomic inequality, genetic factors or a complex interplay of them all is unclear. ${ }^{7}$ Current data are limited in either number of COVID-19 patients, ethnic diversity or event rates with limited adjustment for known risk factors and potential predictors. ${ }^{8-12}$ There is an urgent need for the detailed characterisation of ethnic differences in COVID-19 outcomes and associated risk factors, within diverse populations, to inform practice and policy. Identifying and responding to these ethnic inequalities will be key to mitigating the disproportionate impact of COVID-19 on BAME patients.

Barts Health NHS Trust is the largest NHS trust in the UK, comprising six hospitals; The Royal London Hospital, Newham General Hospital, Whipps Cross Hospital, Mile End Hospital (non-acute), St Bartholomew's Hospital and the London NHS Nightingale Hospital, a purposely built COVID-19 hospital. The hospitals serve the ethnically diverse and socially deprived communities of over 2.6 million people in east London including the London Borough of Newham which experienced 144.3 COVID19-related deaths per 100000 population, ${ }^{13}$ the highest mortality in the UK and Tower Hamlets which has the largest Bangladeshi population in England. ${ }^{14}$ This large, regional dataset afforded extensive analyses of COVID-19 patients of a higher acuity than other studies. We aimed to examine the demographic, socioeconomic, behavioural, biochemical and clinical risk factors associated with outcomes within different ethnic groups of hospitalised COVID-19 patients, using multivariable survival analyses.

\section{METHODS}

\section{Study population}

We considered all patients with confirmed SARS-CoV-2 infection and admitted to the five acute hospitals within Barts Health NHS Trust between 1 January and 13 May 2020. Diagnosis was made using one or more real-time PCR. Those under 16 years were excluded. The first emergency admission encompassing the first positive SARS-CoV-2 test, or the first emergency admission within 2 weeks of positive outpatient testing was defined as the index admission, community diagnoses without an associated emergency hospital admission were excluded. Patients with unknown or undisclosed ethnicity status were collected for comparison but were not included in our primary ethnicity analysis.

\section{Data collection}

Clinical and demographic data, blood results and coding data from current and prior clinical encounters, were collated from the Barts Health Cerner Millennium Electronic Medical Record (EMR) data warehouse and locally held ICNARC databases by members of the direct clinical care team. Mortality data was available to 20 May 2020.

\section{Definition of key variables}

Ethnicity was defined using the NHS ethnic category codes and based on five high-level groups: white, Asian or Asian British, black or black British, mixed and other; to preserve statistical power the mixed and other categories were merged. Relative measures of socioeconomic deprivation were assessed using the English Indices of Deprivation 2020 by matching patient postcode to national Index of Multiple Deprivation (IMD) quintiles using the Office of National Statistics Postcode Directory. ${ }^{15} 16$ Baseline comorbid diseases and Hospital Frailty Risk Score (HFRS) were identified by mapping to ICD-10 coding. ${ }^{17}$ Body mass index (BMI) was calculated by height and weight measurements taken at or during the immediately preceding admission episode. Rockwood Clinical Frailty Scoring (RFS) was assessed by the admitting medical team and recorded in the EMR. ${ }^{18}$ Secondary haemophagocytic lymphohistiocytosis (sHLH) risk score was calculated from peak values of blood results. ${ }^{19}$ Full definitions are detailed in supplementary materials. National Early Warning Score (NEWS) was recorded in the emergency room and general wards by clinical teams in the EMR and is presented as the total score from six physiological parameters. $^{20}$

\section{Outcomes}

The primary outcome was 30-day mortality from time of index COVID-19 hospital admission. Secondary endpoints were 90-day mortality, ICU admission, ICU length of stay, duration of organ support on ICU, need for mechanical ventilation, hospital length of stay and discharge destination if discharged alive from hospital.

\section{Statistical analyses}

A prospective statistical analysis plan was developed. ${ }^{21}$ Baseline characteristics are presented as mean and SD, median and IQR, or number and percentage, as appropriate. We compared proportions using Pearson's $\chi^{2}$ test or Fisher's exact test and continuous variables using twosample t-test or Wilcoxon rank-sum test, as appropriate. Time-to-event analysis was undertaken with follow-up censored at 30 days, survivors with less than 30 days follow-up were censored at time of maximal follow-up. A Cox proportional hazards model was used to assess survival adjusted for age and sex. Age was the only continuous variable. A further multivariable Cox model was developed to assess the effect of predefined risk factors described as associated with adverse outcomes in COVID19: IMD quintile, smoking status, BMI, diabetes, hypertension and chronic kidney disease (CKD). The proportional hazard assumption was assessed by inspection of scaled Schoenfeld residual plots and investigated by stratification. ${ }^{22}$ Logistic regression modelling of ethnicity on ICU treatment using mechanical ventilation was carried out. Effect measures are presented as HR or OR with 95\% 
CI. All analyses were performed using R V.3.6.3 (R Core Team 2020).

\section{Sensitivity analyses}

To assess the effect of including patients with incomplete clinical data, missing data for baseline risk variables included in the multivariable Cox model was imputed using multivariate imputation by chained equations. ${ }^{23}$ Additional multivariable models were also carried out using aggregate Charlson Comorbidity Index (CCI) as a measure of total comorbid disease burden, and HFRS or RFS collected at hospital admission and ABO blood group. Longer-term survival to 90 days was assessed using Cox proportional hazards modelling adjusted for age and sex censored at time of maximal follow-up if survivors had less than 90 days follow-up.

\section{RESULTS}

A total of 1996 patients, aged 16 years and older, with a confirmed SARS-CoV-2 test result with an acute Barts Health admission on or before 13 May 2020 were included in this study (online supplemental figure S1). The recruitment window encompassed the peak time period of COVID-19 diagnoses (online supplemental figure S2). The majority of patients were classified as being in the two most deprived socioeconomic quintiles in England. The ethnic distribution was white $(\mathrm{n}=703$, $35.2 \%)$, Asian or Asian British ( $\mathrm{n}=538,27.0 \%)$, black or Black British ( $\mathrm{n}=340,17.0 \%)$, mixed and other $(\mathrm{n}=156$, $7.8 \%)$ and unknown or undisclosed $(\mathrm{n}=259,13.0 \%)$. Supporting results are detailed in online supplemental file sections S1-S9, online supplemental tables S1-S10, figures S1-S17.

\section{Population characteristics}

Baseline characteristics, interventions and outcomes across ethnic groups are shown in table 1. Black and Asian ethnicity patients were significantly younger with a median age of 59 years (Asian) and 64 years (black), compared with 73 years in the white group $(\mathrm{p}<0.001)$. Comorbidity data were available in $1700(85.2 \%)$ of patients.

Burden of comorbid disease varied between ethnic groups in prevalence, type and age distribution. Overall distribution of COVID-19 risk factors varied with age and ethnicity with diabetes and CKD more prevalent at an earlier age in Asian and black patients and frailty and dementia more prevalent in older white patients (figure 1).

Around one in four patients developed early acute kidney injury (AKI) within 7 days of hospital admission, rates of AKI were highest in the black group (34.7\%). Patients in the black group had higher levels of inflammation $\mathrm{C}$ reactive protein (CRP) (median CRP $181.5 \mathrm{mg} / \mathrm{L}$ ) and fibrinolysis (median D-dimer $2.5 \mathrm{mg} / \mathrm{L}$ ) compared with other ethnicities. As a measure of extent of early physiological derangement NEWS was available in 1443 patients, in comparison to white patients first NEWS was modestly higher in Asian patients (mean 4.2 vs 3.6), $\mathrm{p}=0.001$, but not in black patients (mean 3.7 vs 3.6).

\section{Age-adjusted and sex-adjusted 30-day mortality}

We included 1737 Asian, black and white patients in the primary outcome analysis. Total mortality to 20 May 2020 was $28.7 \%(\mathrm{n}=573)$. Based on the raw data, a greater proportion of white patients died (32.7\%) compared with Asian $(21.1 \%)$ and black $(29.7 \%)$ patients. The majority of deaths $(93.7 \%)$ occurred within 30 days of hospital admission. However, after adjustment for the betweengroup differences in age and sex, patients from Asian and black ethnic groups were at significantly higher risk of death within 30 days compared with white patients (Asian ethnicity (HR 1.49, 95\% CI 1.19 to $1.86, \mathrm{p}<0.001$ ); black patients (HR 1.30, 95\% CI 1.02 to $1.63, \mathrm{p}=0.036$ ). No association was observed in the smaller mixed and other ethnicity group (HR $1.08,95 \%$ CI 0.75 to 1.57 , $\mathrm{p}=0.682$ ) (table 2, figures 2 and 3 ). There was some evidence of non-proportionality for the association between ethnicity and risk of death over time (online supplemental figure S16), consequently these HRs should be interpreted as a weighted average over the 30-day follow-up period. To investigate change in risk over time, we developed an ethnicity-stratified Cox model, this supported the findings of the unstratified model, but suggested that black ethnicity might be associated with a higher early rate of death (online supplemental figure S17).

\section{Multivariable survival modelling}

After inclusion of IMD quintile, smoking history, BMI $\geq 30 \mathrm{~kg} / \mathrm{m}^{2}$, diabetes, hypertension and CKD in a multivariable survival analysis, the association with increased rate of death persisted in Asian patients (HR 1.48, 95\% CI 1.09 to $2.01, \mathrm{p}=0.011 ; \mathrm{n}=1006)$. In black patients, the magnitude of the mortality trend was unchanged, however, was outside the limits of standard statistical significance (HR 1.32, 95\% CI 0.96 to $1.84, \mathrm{p}=0.090$; $\mathrm{n}=1006$ ), potentially due to the smaller sample size. In this model older age, male sex, smoking, BMI $\geq 30 \mathrm{~kg} / \mathrm{m} 2$ and CKD were statistically associated with risk of death (table 3, figures 4 and 5) and there was no statistical evidence that ethnicity violated the proportional hazards assumption. The associations were broadly unchanged when the model was re-fitted after multiple imputation of missing values (online supplemental table S4).

Sensitivity analyses for further multivariable survival models were developed to examine the influence of total comorbidity burden, as assessed by CCI (online supplemental table S5), and measures of frailty, the RFS or HFRS (online supplemental table S6, S7) as well as ABO blood group (online supplemental table S8). In all these analyses, the association between black and Asian ethnicity and 30-day mortality remained significant. Adjusting for RFS raised the odds of 30-day mortality to an HR of 1.98 (95\% CI 1.37 to 2.86; $\mathrm{p}<0.001$ ) in Asian groups and to a HR of 1.67 (95\% CI 1.14 to 2.45; $\mathrm{p}=0.009$ ) in 
Table 1 Study population baseline characteristics stratified by ethnic group, $\mathrm{n}(\%)$ unless otherwise stated

\begin{tabular}{|c|c|c|c|c|c|c|}
\hline \multirow[b]{3}{*}{$\mathbf{n}$} & \multicolumn{5}{|c|}{ Stratified by ethnic group } & \multirow[b]{3}{*}{$P$ value } \\
\hline & $\begin{array}{l}\text { Asian or Asian } \\
\text { British }\end{array}$ & $\begin{array}{l}\text { Black or Black } \\
\text { British }\end{array}$ & $\begin{array}{l}\text { Mixed and other } \\
\text { ethnic groups }\end{array}$ & White & $\begin{array}{l}\text { Unknown and } \\
\text { undisclosed }\end{array}$ & \\
\hline & 538 & 340 & 156 & 703 & 259 & \\
\hline Age (years), mean (SD) & $57.8(18.5)$ & $64.2(16.9)$ & $59.5(17.2)$ & $69.4(17.7)$ & $59.8(16.5)$ & $<0.001$ \\
\hline Age (years), median (IQR) & $59.0(44.0-71.0)$ & $64.0(53.0-79.0)$ & $59.0(47.8-72.3)$ & $73.0(58.0-84.0)$ & $61.0(50.0-71.5)$ & $<0.001$ \\
\hline Male & $332(61.7)$ & $193(56.8)$ & $103(66.0)$ & $404(57.5)$ & $178(68.7)$ & 0.01 \\
\hline 1 (most deprived) & $139(26.0)$ & $124(36.7)$ & $50(32.9)$ & $183(26.2)$ & $66(25.7)$ & \\
\hline 2 & $291(54.5)$ & $165(48.8)$ & $72(47.4)$ & $269(38.5)$ & $124(48.2)$ & \\
\hline 3 & $49(9.2)$ & $34(10.1)$ & $20(13.2)$ & $99(14.2)$ & $44(17.1)$ & \\
\hline 4 & $35(6.6)$ & $9(2.7)$ & $7(4.6)$ & $86(12.3)$ & $18(7.0)$ & \\
\hline 5 (least deprived) & $20(3.7)$ & $6(1.8)$ & $3(2.0)$ & $62(8.9)$ & $5(1.9)$ & \\
\hline By category & & & & & & 0.04 \\
\hline$<18.5 \mathrm{~kg} / \mathrm{m}^{2}$ & $9(2.8)$ & $8(3.6)$ & $1(1.3)$ & $34(6.9)$ & $11(8.5)$ & \\
\hline 18.5 to $<25 \mathrm{~kg} / \mathrm{m}^{2}$ & $101(31.2)$ & $57(25.3)$ & $31(40.3)$ & $160(32.5)$ & $43(33.1)$ & \\
\hline $25-<30 \mathrm{~kg} / \mathrm{m}^{2}$ & $114(35.2)$ & $83(36.9)$ & $27(35.1)$ & $145(29.5)$ & $40(30.8)$ & \\
\hline 30 to $<40 \mathrm{~kg} / \mathrm{m}^{2}$ & 87 (26.9) & 65 (28.9) & $17(22.1)$ & $126(25.6)$ & $28(21.5)$ & \\
\hline$\geq 40 \mathrm{~kg} / \mathrm{m}^{2}$ & $13(4.0)$ & $12(5.3)$ & $1(1.3)$ & 27 (5.5) & $8(6.2)$ & \\
\hline \multicolumn{7}{|l|}{ Comorbidity $(n=1700)$} \\
\hline Obesity & $108(23.6)$ & $82(27.9)$ & $18(14.9)$ & $161(26.2)$ & $40(18.7)$ & 0.01 \\
\hline Ischaemic heart disease & $102(22.3)$ & $62(21.1)$ & $12(9.9)$ & $149(24.3)$ & $21(9.8)$ & $<0.001$ \\
\hline $\begin{array}{l}\text { Chronic obstructive } \\
\text { pulmonary disease }\end{array}$ & $119(26.0)$ & $45(15.3)$ & $18(14.9)$ & $181(29.5)$ & $34(15.9)$ & $<0.001$ \\
\hline Diabetes & $220(48.1)$ & $157(53.4)$ & $49(40.5)$ & $179(29.2)$ & $59(27.6)$ & $<0.001$ \\
\hline HTN & $261(57.1)$ & $212(72.1)$ & $64(52.9)$ & $376(61.2)$ & $96(44.9)$ & $<0.001$ \\
\hline Moderate to severe CKD & $92(20.1)$ & $93(31.6)$ & $16(13.2)$ & $145(23.6)$ & $17(7.9)$ & $<0.001$ \\
\hline End-stage renal disease & $39(8.5)$ & $36(12.2)$ & $7(5.8)$ & $27(4.4)$ & $4(1.9)$ & $<0.001$ \\
\hline Liver disease & $49(9.1)$ & $24(7.1)$ & $12(7.7)$ & $58(8.3)$ & $12(4.6)$ & 0.25 \\
\hline Cancer & $30(6.6)$ & $26(8.8)$ & $8(6.6)$ & $68(11.1)$ & $12(5.6)$ & 0.04 \\
\hline Cancer with metastases & $8(1.8)$ & $5(1.7)$ & $1(0.8)$ & $22(3.6)$ & $6(2.8)$ & 0.18 \\
\hline AIDS & $0(0.0)$ & $5(1.7)$ & $0(0.0)$ & $1(0.2)$ & $0(0.0)$ & 0.001 \\
\hline $\begin{array}{l}\text { Charlson Comorbidity Index } \\
(n=1700)\end{array}$ & & & & & & $<0.001$ \\
\hline 0 & $131(28.7)$ & $66(22.4)$ & $42(34.7)$ & $143(23.3)$ & $91(42.5)$ & \\
\hline $1-2$ & $178(38.9)$ & $100(34.0)$ & $50(41.3)$ & $203(33.1)$ & $88(41.1)$ & \\
\hline $3-4$ & $70(15.3)$ & $52(17.7)$ & $16(13.2)$ & $146(23.8)$ & $20(9.3)$ & \\
\hline$\geq 5$ & $78(17.1)$ & $76(25.9)$ & $13(10.7)$ & $122(19.9)$ & $15(7.0)$ & \\
\hline $\begin{array}{l}\text { Rockwood Frailty Score } \\
(n=831)\end{array}$ & & & & & & $<0.001$ \\
\hline 1-2 (very fit, well) & $31(15.9)$ & $6(4.3)$ & 7 (14.9) & $36(9.7)$ & $15(18.8)$ & \\
\hline
\end{tabular}


Table 1 Continued

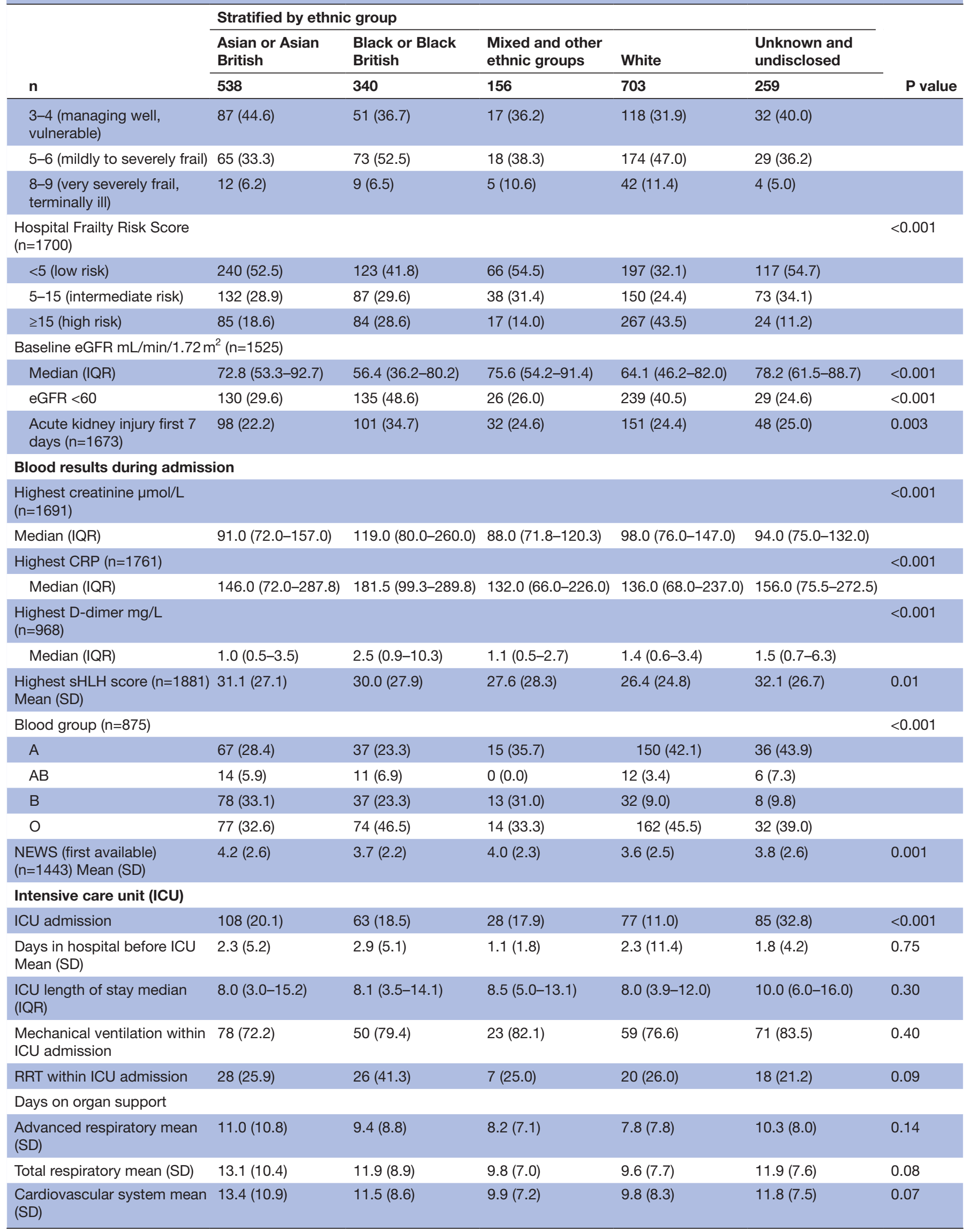


Table 1 Continued

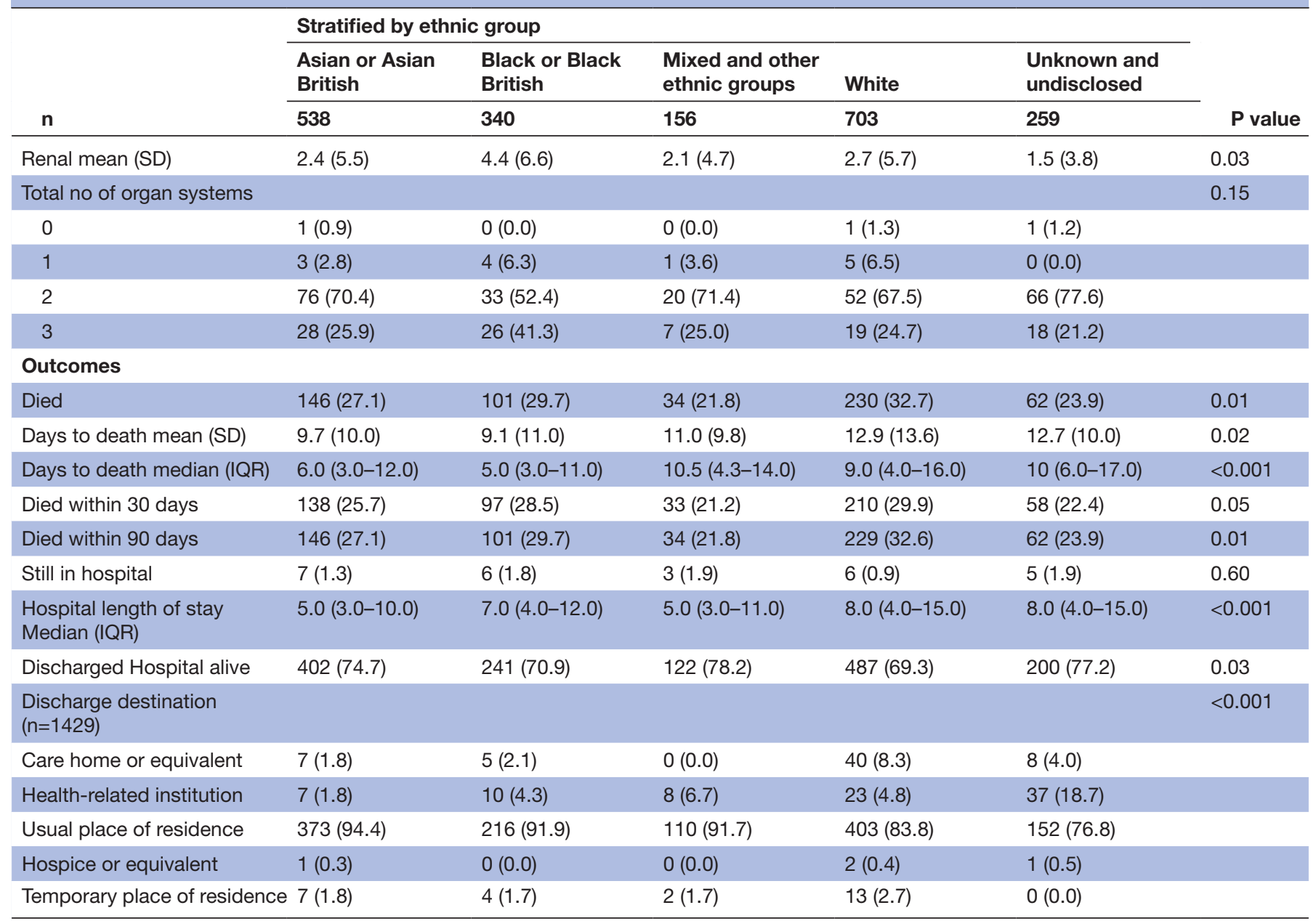

Total $\mathrm{n}=1996$ unless otherwise stated.

$\mathrm{P}$ values based on $\mathrm{X}^{2}$ (for categorical) or Kruskal-Wallis test (for continuous).

BMI, body mass index; CKD, chronic kidney disease; CRP, C reactive protein; HTN, hypertension; ICU, intensive care unit; IMD, Index of Multiple Deprivation; NEWS, National Early Warning Score; RRT, renal replacement therapy; sHLH, secondary haemophagocytic lymphohistiocytosis (without known underlying immunosuppression and bone marrow aspirate data); TIA, transient ischaemic accident.

black groups, with similar effect size in analysis adjusted for the HFRS. After inclusion of ABO blood grouping in and age-adjusted and sex-adjusted multivariable model risks of death in Asian, black and mixed and other ethnic groups was increased. Asian ethnicity also continued to be associated with greater risks of death through to 90 days follow-up (HR 1.46, 95\% CI 1.18 to $1.81, \mathrm{p}<0.001$; $\mathrm{n}=1737$ ) (online supplemental table S9).

\section{Critical care-related outcomes}

In the white group, $11.0 \%$ of patients were admitted to ICU compared with $20.1 \%$ of the Asian group and $18.5 \%$ of the black group $(p<0.001)$. In those admitted to ICU, rates of mechanical ventilation requiring intubation did not differ significantly by ethnicity at $76.6 \%$ in the white group, $72.2 \%$ in the Asian group and $79.4 \%$ in the black group. Similarly, while rates of ICU admission differed significantly between ethnic groups, time from hospital to ICU admission and length of ICU stay did not. Across the entire hospitalised cohort Asian (OR 1.54, 95\% CI 1.06 to
2.23, $\mathrm{p}=0.023 ; \mathrm{n}=1737)$ and black (OR $1.80,95 \%$ CI 1.20 to $2.71, \mathrm{p}=0.005 ; \mathrm{n}=1737$ ) ethnicities were associated with increased age-adjusted and sex-adjusted risk of receiving invasive mechanical ventilation in ICU (online supplemental table S10). There was a trend towards increased renal replacement therapy use in black patients $(41.3 \%)$ admitted to ICU compared with $20 \%-25 \%$ across other ethnic groups $(\mathrm{p}=0.09)$.

\section{DISCUSSION}

We report on treatment and outcomes in COVID-19 patients hospitalised in East London throughout the peak of the UK pandemic, a population with the UK's highest COVID-19 mortality. To our knowledge, this is one of the largest UK hospital COVID-19 cohorts reported, and certainly the most diverse, with only $35.2 \%$ of 1996 patients identified as White ethnicity. We found those of Asian ethnicity to be at the highest risk of death within 30 


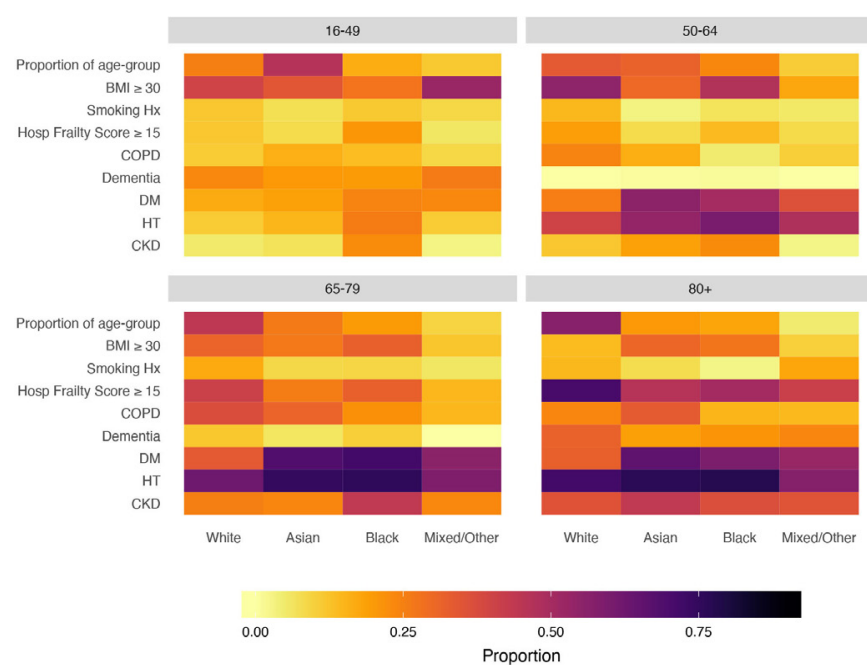

Figure 1 Heat map of prognostic factors in COVID-19 hospital admissions by age and ethnic background showing proportions within each ethnic group for each age group. Asian and black patients differed from those of white background in the presence of risk factors and their age distribution, however, differences were also apparent between different black and minority ethnic groups at different ages. Proportions are of those with data (see table 1). BMI, body mass index; CKD, chronic kidney disease; COPD, chronic obstructive pulmonary disease; DM, diabetes mellitus; $\mathrm{HT}$, hypertension.

days (HR 1.49, 95\% CI 1.19 to $1.86, \mathrm{p}<0.001$ ), a finding that persisted at 90 days. Risk of death in black patients was also greater than those of White ethnicity (HR 1.30, 95\% CI 1.02 to $1.63, \mathrm{p}=0.036$ ). This disparity extended to need for ICU care with Asian and black patients experiencing a $50 \%-80 \%$ increased risk of receiving mechanical ventilation in ICU compared with white patients of a similar age.

Table 2 Association of ethnic group with mortality to 30 days using Cox proportional hazards modelling, age and sex corrected

\begin{tabular}{|c|c|c|c|c|}
\hline & \multicolumn{2}{|l|}{$\mathbf{n}$} & \multirow{2}{*}{$\begin{array}{l}\text { Unadjusted } \\
\text { HR }(95 \% \mathrm{Cl})\end{array}$} & \multirow[b]{2}{*}{$P$ value } \\
\hline & Total & Events & & \\
\hline $\begin{array}{l}\text { Age (25th vs } \\
75 \text { th centile) }\end{array}$ & - & - & $\begin{array}{l}4.50 \text { (3.74 to } \\
5.42)\end{array}$ & $<0.0001$ \\
\hline Sex (male) & - & - & $\begin{array}{l}1.55 \text { (1.28 to } \\
1.87)\end{array}$ & $<0.0001$ \\
\hline \multicolumn{5}{|l|}{ Ethnic group } \\
\hline $\begin{array}{l}\text { Asian or } \\
\text { Asian British }\end{array}$ & 521 & 134 & $\begin{array}{l}1.49(1.19 \text { to } \\
1.86)\end{array}$ & $<0.001$ \\
\hline $\begin{array}{l}\text { Black or } \\
\text { black British }\end{array}$ & 331 & 94 & $\begin{array}{l}1.30(1.02 \text { to } \\
1.65)\end{array}$ & 0.036 \\
\hline $\begin{array}{l}\text { Mixed and } \\
\text { other ethnic } \\
\text { groups }\end{array}$ & 150 & 34 & $\begin{array}{l}1.08 \text { (0.75 to } \\
1.57)\end{array}$ & 0.682 \\
\hline White & 674 & 206 & Reference & - \\
\hline
\end{tabular}

Censored to 30 days follow-up, observations 1737, events 478 .

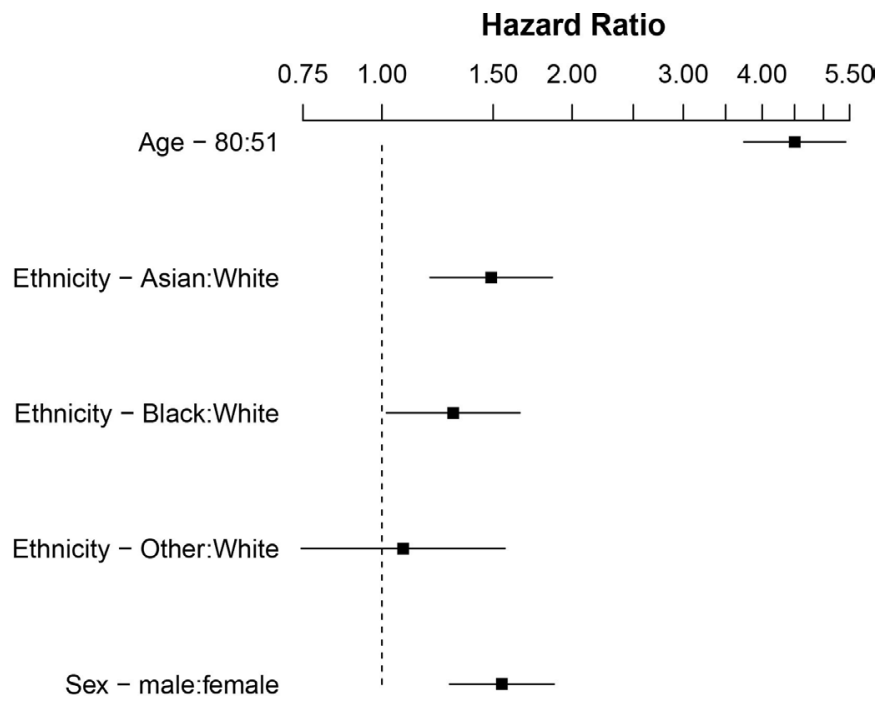

Figure 2 Forest plot showing HRs of mortality to 30 days comparing ethnic groups, age and sex corrected, on log scale.

\section{Strengths and limitations}

We believe this study is both one of the largest and most detailed of studies exploring COVID-19 outcomes in BAME populations so far reported. In contrast to many previous studies examining ethnicity and COVID-19 outcomes, we were able to address the contributions of socioeconomic deprivation, comorbid disease, premorbid function, lifestyle and demographic factors to ethnic disparities in COVID-19 outcomes, including ICU interventions. Our analysis was strengthened by the inclusion of measures of frailty which is a critical determinant of outcomes in acute

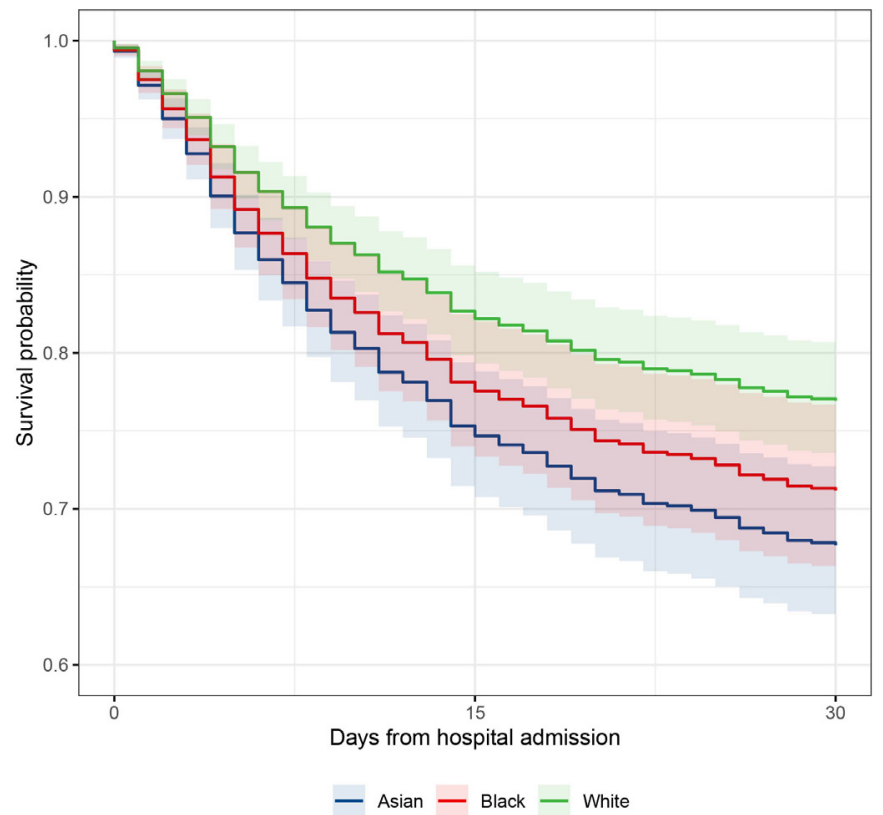

Figure 3 Survival curve to 30 days comparing predicted survival of Asian, black and white ethnic groups (mixed and other group omitted for clarity), in an age and sex adjusted Cox hazard analysis. Survival curves adjusted to median age 65 years and male sex. 
Table 3 Multivariable analysis of mortality to 30 days using Cox proportional hazards modelling, age and sex corrected

\begin{tabular}{|c|c|c|}
\hline & \multicolumn{2}{|l|}{ Adjusted } \\
\hline & HR (95\% Cl) & $P$ value \\
\hline Age (25th vs 75 th centile) & 3.24 (2.46 to 4.26$)$ & $<0.0001$ \\
\hline Sex (male) & 1.47 (1.15 to 1.88$)$ & 0.002 \\
\hline \multicolumn{3}{|l|}{ Ethnic group } \\
\hline Asian or Asian British & 1.48 (1.09 to 2.01$)$ & 0.011 \\
\hline Black or black British & 1.32 (0.96 to 1.84$)$ & 0.090 \\
\hline $\begin{array}{l}\text { Mixed and other ethnic } \\
\text { groups }\end{array}$ & 0.90 (0.49 to 1.65$)$ & 0.733 \\
\hline White & Reference & - \\
\hline \multicolumn{3}{|l|}{ IMD quintile } \\
\hline 1 (most deprived) & 0.79 (0.55 to 1.14$)$ & 0.213 \\
\hline 2 & 0.79 (0.54 to 1.15$)$ & 0.218 \\
\hline 3 & 0.88 (0.61 to 1.27$)$ & 0.503 \\
\hline 4 & 0.77 (0.53 to 1.12$)$ & 0.176 \\
\hline 5 (least deprived) & Reference & - \\
\hline Smoking & 1.56 (1.13 to 2.17$)$ & 0.008 \\
\hline $\mathrm{BMI} \geq 30 \mathrm{~kg} / \mathrm{m}^{2}$ & $1.42(1.09$ to 1.85$)$ & 0.009 \\
\hline Diabetes & 1.29 (1.00 to 1.67$)$ & 0.055 \\
\hline HTN & $1.32(0.92$ to 1.89$)$ & 0.131 \\
\hline CKD & 1.34 (1.04 to 1.73$)$ & 0.023 \\
\hline
\end{tabular}

Variables included IMD quintile, smoking, BMI $\geq 30 \mathrm{~kg} / \mathrm{m}^{2}$, diabetes. Censored to 30 days follow up, observations 1006, events 281 . BMI, body mass index; CKD, chronic kidney disease; HTN, hypertension; IMD, Index of Multiple Deprivation.

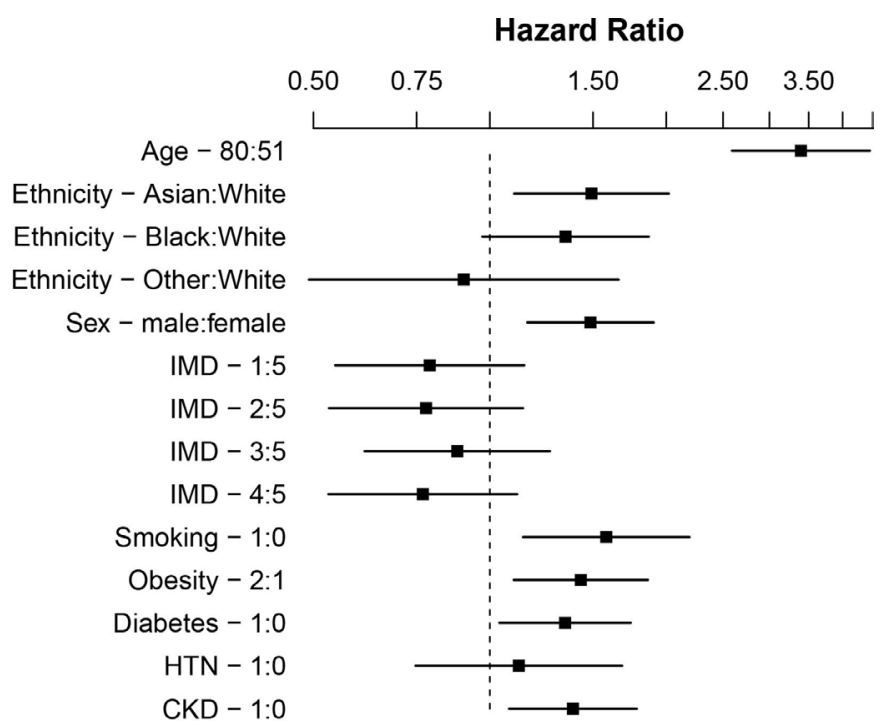

Figure 4 Forest plot showing HRs of mortality to 30 days comparing ethnic groups, age and sex corrected, on log scale. Additional variables included Index of Multiple Deprivation (IMD) quintile (five least deprived), smoking, body mass index $\geq 30 \mathrm{~kg} / \mathrm{m}^{2}$, diabetes. CKD, chronic kidney disease; HTN, hypertension.

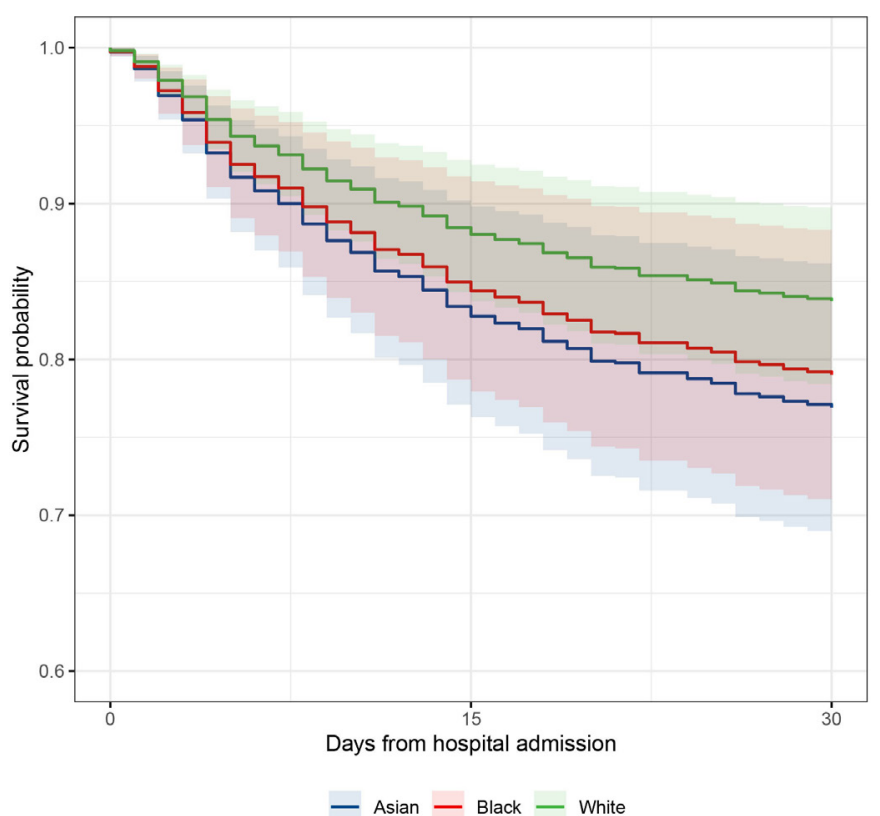

Figure 5 Survival curve to 30 days from multivariable analysis comparing Asian, black and white ethnic groups. Survival modelled for median age 65 years and male sex, Index of Multiple Deprivation (IMD) least deprived quintile, no history of baseline risk factors defined as non-smoking, BMI $<30 \mathrm{~kg} / \mathrm{m}^{2}$ and no diabetes, hypertension or chronic kidney disease. Statistically significant difference in survival between Asian group and white group persists after adjustment for age, sex, social deprivation and major COVID-19 risk factors. BMI, body mass index.

disease as well as a potential driver of clinician decision making. It should be acknowledged, however, that frailty has social and biological dimensions and measures have not been extensively validated in BAME groups.

Importantly, this study was conducted in a single region where COVID-19 has had significant impact, and thus, is not confounded by differences in incidence of COVID-19 disease across the UK, regional concentration of minority ethnic groups and regional differences in the time course of the epidemic. In addition, we employed a prespecified statistical analysis plan and performed multiple sensitivity analyses to test the robustness of our findings.

Limitations in our analyses must also be considered. Importantly, SARS-CoV-2 testing has an appreciable false negative rate and suspected, but not proven, cases are an important group. Nevertheless, given that clinical suspicion varied both between cases and across the time course of the epidemic with coding of suspected cases being inconsistent, in line with the vast majority of published COVID-19 analyses, we only included proven COVID-19 cases. Testing was available for all hospitalised patients with suspected COVID-19 disease, so availability of testing was not a bias. However, suspected diagnoses should be considered in future studies, particularly those occurring outside of hospitals, where not all clinical diagnoses may have been tested.

Similar to many hospital datasets there were missing data for a proportion of covariates, ${ }^{89}$ however, $85 \%$ of 
patients had coding data for assessment of comorbidity and $63 \%$ measured height and weight data, providing a large sample with detailed data for analysis. We also imputed missing data and performed sensitivity analyses on our multivariable comorbidity models. This reinforced the observed ethnic differences, providing further confidence that our findings were not affected by missing data.

Like many datasets, our ethnic categorisations were aggregated and did not reflect the vast heterogeneity within ethnic categories (such as Bangladeshi, Pakistani, black African or black Caribbean). Indeed, the descriptive term 'BAME' itself is particularly crude and we recognise its limitation. Despite its size, our study lacked the power to assess a more detailed ethnicity breakdown. In addition, our observations in those of Asian ethnicity are likely skewed by our large Bangladeshi community, which has specific socioeconomic and healthcare inequalities. It is, therefore, important that, suitably powered, analyses are conducted to expose differences between subethnic categories. Similarly, while we have explored socioeconomic factors, our analysis does not allow us to contextualise a number of potential sociospatial factors including household composition, environmental factors and occupation. These should be considered in future research.

\section{Comparison with other studies}

Our findings differ from predominant reports in the UK and USA in which black ethnicity has been consistently associated with greater COVID-19-related mortality. ${ }^{624}$ Preliminary analyses of the UK ICNARC report on COVID-19 in critical care highlighted black ethnicity with the highest likelihood of being admitted to intensive care compared with a matched population $(10.7 \%$ vs $6.5 \%) .{ }^{25}$ Similarly, in a large UK primary care linked cohort, black patients were also to found to be at highest risk of COVID-19related death. ${ }^{9}$ In a US study, the composite relative risk of COVID-related death compared with white ethnicity was 3.57 in black populations, and 1.88 for Latinos. ${ }^{24}$ Our findings suggest specific South Asian communities may have at least the same or higher risk in COVID-19 as those of black background. This may reflect characteristics of the large South Asian, and specifically Bangladeshi, community in East London, poorly represented in other studies. Recently the ISARIC CCP-UK investigators have described association of ethnicity and outcome in a very large cohort of UK patients, finding Asian, but not Black background was associated with increased risk of death in confirmed or suspected COVID-19. ${ }^{26}$ While this study documented up to $40 \%$ of UK COVID-19 cases, it represented a selection from the total COVID-19 population from across the UK, and, at least in terms of ICU cases, ethnic minorities were significantly under-represented compared with the English ICU COVID-19 population. In contrast while smaller, this study focused on an unbiased population comprising all hospitalised patients in a single geographical area with a much higher level of ethnic diversity. Consequently, we feel our analysis complements
ISARIC CCP-UK and provides greater clinical detail in a regionally homogenous population.

\section{Potential confounding associations with risk of death in COVID-19}

Older age has been significantly associated with increased COVID-19 mortality across a range of studies. ${ }^{2-4}$ In our cohort, patients from Asian and black backgrounds were strikingly younger than White patients. However, despite the expected protective factor of younger age, when this was accounted for, those from black and Asian backgrounds were more likely to die. The prevalence of comorbid disease has been well described as a risk factor for COVID-19 disease and death. ${ }^{34}$ We found different ethnic groups had differing age distribution of baseline comorbidities such as hypertension, diabetes, chronic obstructive pulmonary disease and dementia. Despite accounting for these and other described predictors of poor outcomes, increased risk of death in Asian and black populations was not attenuated, suggesting comorbidities are not the sole drivers of ethnicity-associated risk.

$\mathrm{ABO}$ blood group has recently been suggested to affect the risk of symptomatic COVID-19 and need for respiratory support with supplemental oxygen. ${ }^{12}{ }^{27}$ In these analyses, blood group $\mathrm{O}$ was associated with less disease acquisition than group A. As there are well-described differences in blood group distribution with ethnicity (in particular, prevalence of blood group B in Asian and to a lesser extent black populations), in a post hoc analysis, we assessed the association between $\mathrm{ABO}$ group and risk of death in 875 patients with blood group data. In contrast to studies focused on risk of COVID-19 acquisition in our cohort of hospitalised COVID-19 diagnoses, blood group $\mathrm{O}$ was associated with higher risk of death and blood group B the lowest. Accordingly, when we included $\mathrm{ABO}$ blood group in a multivariable survival analysis with age, sex the association between black and Asian background and increased risk of death was not attenuated but magnified. This suggests ethnic imbalances in blood group distribution did not explain the mortality associations observed in our population.

Patients identified as frail have been predicted to have worse COVID-19-related health outcomes, ${ }^{28}$ and lower likelihood of benefiting from complex acute interventions, including critical care. In this study, white patients, in addition to being notably older than other ethnicities, had higher degrees of frailty. Accounting for measures of frailty magnified the association seen between Asian and black ethnicity and death. This suggests that while in white patients COVID-19-related death may have occurred in already frail and functionally vulnerable patients, in both Asian and black patients, COVID-19-related deaths are likely to be occurring prematurely, in younger, fitter individuals with less functional vulnerability.

In our cohort, all ethnic groups experienced high levels of deprivation, however, worse deprivation was not associated with higher likelihood of mortality, suggesting 
ethnicity may affect outcomes independent of purely geographical and socioeconomic factors. ${ }^{29}$

We found evidence for worse disease severity in black and Asian groups as evidenced by higher rates of ICU admission and higher rates of AKI, and high levels of D-dimers and CRP in black patients. High CRP and D-dimer levels have been identified as important inflammatory markers which strongly correlate with COVID-19 disease severity and prognosis. ${ }^{30}$ Our data suggest potential biological differences in host response to COVID-19 may occur between ethnicities, however, causative associations in determining COVID-19-related mortality have not been demonstrated.

Finally, although COVID-19 has cast the effects of ethnic inequalities on health outcomes into sharp focus, these inequalities are not new. Health inequalities within and between ethnic minority groups are widely documented and the effects of structural racism are transmitted across generations. ${ }^{31}$ The risk factors already discussed such comorbidity and obesity are speculated to intersect and be inextricably linked with wider social determinants such as poor living conditions, key worker roles and language barriers which impede the adoption of preventative measures. ${ }^{29} 3233$ Some researchers have postulated that ethnic inequalities may be associated with decreased symptom recognition and poor engagement with health services. ${ }^{34}$ However, while frequency of ICU admission, AKI and need for mechanical ventilation suggests more severe peak disease in minority ethnic groups, time to ICU admission did not differ and differences in first total NEWS were at most modest, suggesting against a large effect from delayed presentation.

\section{CONCLUSION}

In this analysis of a large, ethnically diverse and socioeconomically challenged cohort, hospitalised patients of Asian and black background with COVID-19 were at increased risk of premature death, independent of frailty, comorbidities and social deprivation. Failure to robustly respond to the ethnic disparities so conspicuously unmasked during the COVID-19 pandemic can only further entrench and inflict them on future generations.

\section{Author affiliations}

${ }^{1}$ Blizard Institute, Queen Mary University of London, London, UK, E1 2AT

${ }^{2}$ Sexual Health and HIV Medicine, Barts Health NHS Trust, London, UK, E1 1FR

${ }^{3}$ William Harvey Research Institute, Queen Mary University of London, London, UK, EC1M 6BQ

${ }^{4}$ Adult Critical Care Unit, Barts Health NHS Trust, London, UK, E1 1FR

Acknowledgements We would like to acknowledge the assistance of the ICU clinical leads and ICU audit teams at the individual sites for access to local audit data: Gail Marshall (RLH and NGH), Susan Kolakottu Thomas (NUH), Jonathan Barry (SBH), John Sant (WXH). JP would like to thank Dr Bhavi Trivedi, Barts Health Clinical Analytics Officer for her past and current advice on data extraction from Trust EMR systems. More widely we would like to acknowledge the courage and commitment of all staff members of Barts Health NHS Trust during the COVID-19 epidemic and to extent our condolences to all affected by death or serious illness related to COVID-19. The strength and character of the East London community from every background has once again been demonstrated.
Contributors VJA developed the study concept, designed the study, wrote the study protocol, submitted the ethics application, provided critical review of the findings and wrote the manuscript. YIW wrote the statistical analysis plan, performed data extraction, performed statistical analysis, provided critical review of the findings and wrote the manuscript. RD developed the study concept, designed the study, provided critical review of the findings and wrote the manuscript. ZAP provided critical review of the findings and wrote the manuscript. RMP developed the study concept, designed the study, provided critical review of the findings and wrote the manuscript. CMO developed the study concept, designed the study, provided critical review of the findings and wrote the manuscript. JRP developed the study concept, designed the study, wrote the study protocol, submitted the ethics application, performed data extraction, performed statistical analysis, provided critical review of the findings and wrote the manuscript. All authors approved the final version of the manuscript. The data were collated and analysed on behalf of all clinicians at Barts Health.

Funding The authors have not declared a specific grant for this research from any funding agency in the public, commercial or not-for-profit sectors.

Competing interests All authors have completed the ICMJE uniform disclosure form at www.icmje.org/coi_disclosure.pdf and declare: no support from any organisation for the submitted work; no financial relationships with any organisations that might have an interest in the submitted work in the previous 3 years; no other relationships or activities that could appear to have influenced the submitted work.

Patient consent for publication Not required.

Ethics approval This study was approved by HRA and Yorkshire \& The Humber - Bradford Leeds Research Ethics Committee (Ethics reference 20/YH/0159). The study was sponsored by Barts Health NHS Trust.

Provenance and peer review Not commissioned; externally peer reviewed.

Data availability statement No data are available. The statistical analysis plan can be accessed online. The authors will be happy to consider additional analyses of the anonymised dataset on request. The need for stringent measures to prevent reidentification of individuals within a discrete geographical location and limited time period, however, preclude sharing of patient level dataset in a GDPR compliant form.

Supplemental material This content has been supplied by the author(s). It has not been vetted by BMJ Publishing Group Limited (BMJ) and may not have been peer-reviewed. Any opinions or recommendations discussed are solely those of the author(s) and are not endorsed by BMJ. BMJ disclaims all liability and responsibility arising from any reliance placed on the content. Where the content includes any translated material, BMJ does not warrant the accuracy and reliability of the translations (including but not limited to local regulations, clinical guidelines, terminology, drug names and drug dosages), and is not responsible for any error and/or omissions arising from translation and adaptation or otherwise.

Open access This is an open access article distributed in accordance with the Creative Commons Attribution Non Commercial (CC BY-NC 4.0) license, which permits others to distribute, remix, adapt, build upon this work non-commercially, and license their derivative works on different terms, provided the original work is properly cited, appropriate credit is given, any changes made indicated, and the use is non-commercial. See: http://creativecommons.org/licenses/by-nc/4.0/.

ORCID iD

Yize I Wan http://orcid.org/0000-0001-6445-8991

\section{REFERENCES}

1 Eurosurveillance Editorial Team. Updated rapid risk assessment from ECDC on the novel coronavirus disease 2019 (COVID-19) pandemic: increased transmission in the EU/EEA and the UK. Eurosurveillance 2020;25.

2 Wang D, Hu B, Hu C, et al. Clinical characteristics of 138 hospitalized patients with 2019 novel coronavirus-infected pneumonia in Wuhan, China. JAMA 2020;323:1061-9.

$3 \mathrm{~W}-\mathrm{j}$ G, W-h L, J-x H. Cardiovascular comorbidity and its impact on patients with Covid-19. Europ Resp J 2020;2001227.

4 Xu X-W, Wu X-X, Jiang X-G, et al. Clinical findings in a group of patients infected with the 2019 novel coronavirus (SARSCov-2) outside of Wuhan, China: retrospective case series. BMJ 2020;368:m606. 
5 Intensive Care National Audit and Research Centre. ICNARC report on COVID-19 in critical care 29 May 2020. London: Intensive Care National Audit and Research Centre.

6 Platt L, Warwick R. Are some ethnic groups more vulnerable to COVID-19 than others? Institute of Fiscal Studies, 2020.

7 Khunti K, Singh AK, Pareek M, et al. Is ethnicity linked to incidence or outcomes of covid-19? BMJ 2020;369:m1548.

8 Perez-Guzman PN, Daunt A, Mukherjee S. Clinical characteristics and predictors of outcomes of hospitalised patients with COVID-19 in a London NHS trust: a retrospective cohort study (29-04-2020) 2020.

9 Williamson E, Walker AJ, Bhaskaran KJ, et al. OpenSAFELY: factors associated with COVID-19-related Hospital death in the linked electronic health records of 17 million adult NHS patients. Cold Spring Harbor Laboratory 2020.

10 Aldridge RW, Lewer D, Katikireddi SV, et al. Black, Asian and minority ethnic groups in England are at increased risk of death from COVID-19: indirect standardisation of NHS mortality data. Wellcome Open Res 2020;5:88

11 de Lusignan S, Dorward J, Correa A, et al. Risk factors for SARS-CoV-2 among patients in the Oxford Royal College of general practitioners research and surveillance centre primary care network: a cross-sectional study. Lancet Infect Dis 2020;20:1034-42.

12 , Ellinghaus D, Degenhardt F, et al, Severe Covid-19 GWAS Group. Genomewide association study of severe Covid-19 with respiratory failure. N Engl J Med 2020;383:1522-34.

13 Office For National Statistics. Deaths involving COVID-19 by local area and socioeconomic deprivation: deaths occurring between 1 March and 17 April 2020, 2020.

14 Office for National Statistics. 2011 census: aggregate data data collection. 2020. UK Data Service, 2020.

15 English indices of deprivation 2019. Available: https://www.gov.uk/ government/statistics/english-indices-of-deprivation-2019 [Accessed 1 May 2020].

16 ONS postcode directory (February 2020). Available: https://geoportal. statistics.gov.uk/datasets/ons-postcode-directory-february-2020 [Accessed cited 1st May 2020].

17 Gilbert T, Neuburger J, Kraindler J, et al. Development and validation of a hospital frailty risk score focusing on older people in acute care settings using electronic Hospital records: an observational study. Lancet 2018;391:1775-82.
18 Rockwood K, Song X, MacKnight C, et al. A global clinical measure of fitness and frailty in elderly people. CMAJ 2005;173:489-95.

19 Mehta P, McAuley DF, Brown M, et al. COVID-19: consider cytokine storm syndromes and immunosuppression. Lancet 2020;395:1033-4.

20 RCP. National early warning score (news) 2: standardising the assessment of acute-illness severity in the NHS. updated report of a working party. London, 2017.

21 ETHICAL: Ethnic disparities in COVID-19 admissions in East London. Available: https://qmro.qmul.ac.uk/xmlui/handle/123456789/64155

22 Stensrud MJ, Hernán MA. Why test for proportional hazards? JAMA 2020;323:1401

23 Sv B, Groothuis-Oudshoorn K. mice: multivariate imputation by Chained equations inR. J Statist Software 2011;45.

24 Gross CP, Essien UR, Pasha S. Racial and ethnic disparities in population level Covid-19 mortality. medRxiv 2020:20094250.

25 Intensive Care National Audit and Research Centre. ICNARC report on COVID-19 in critical care - 10 Apr 2020.

$26 \mathrm{EMaD} \mathrm{H}$, Annemarie B. Ethnicity and outcomes from COVID-19: the ISARIC CCP-UK prospective observational cohort study of hospitalised patients, 2020. Available: https://ssrn.com/abstract= 3618215 or http://dx.doi.org/10.2139/ssrn.3618215

27 Zietz M, Zucker J, Tatonetti NP. Testing the association between blood type and COVID-19 infection, intubation, and death. medRxiv 2020 doi:10.1101/2020.04.08.20058073

28 Hubbard RE, Maier AB, Hilmer SN, et al. Frailty in the face of COVID-19. Age Ageing 2020;49:499-500.

29 Public Health England. Disparities in the risk and outcomes of COVID-19, 2020

30 Zhou Y, Yang Z, Guo Y. A new predictor of disease severity in patients with COVID-19 in Wuhan, China. medRxiv 2020:20042119.

31 Nazroo JY The structuring of ethnic inequalities in health: economic position, racial discrimination, and racism. Am J Public Health 2003;93:277-84

32 Yancy CW. COVID-19 and African Americans. JAMA 2020;323:1891.

33 Atchison CJ, Bowman L, Vrinten C, et al. Perceptions and behavioural responses of the general public during the COVID-19 pandemic: a cross-sectional survey of UK adults. cold Spring Harbor laboratory 2020.

34 Wechkunanukul K, Grantham H, Damarell R, et al. The association between ethnicity and delay in seeking medical care for chest pain: a systematic review. JBI Database System Rev Implement Rep 2016;14:208-35. 